\title{
Fear Load: The Psychophysiological Over-expression of Fear as an Intermediate Phenotype Associated with Trauma Reactions
}

Seth Davin Norrholm ${ }^{1,2}$, Ebony M. Glover ${ }^{3}$, Jennifer S. Stevens ${ }^{2}$, Negar Fani ${ }^{2}$, Isaac R. Galatzer-

Levy $^{4}$, Bekh Bradley ${ }^{1,2}$, Kerry J. Ressler ${ }^{5,6}$, and Tanja Jovanovic ${ }^{2}$

${ }^{1}$ Atlanta Veterans Affairs Medical Center, Mental Health Service Line, Decatur, GA

${ }^{2}$ Department of Psychiatry and Behavioral Sciences, Emory University School of Medicine, Atlanta, GA

${ }^{3}$ Department of Psychology, Kennesaw State University, Kennesaw, GA

${ }^{4}$ Departments of Psychiatry, NYU School of Medicine, New York, NY

${ }^{5}$ Howard Hughes Medical Institute, Bethesda, MD

${ }^{6}$ Yerkes National Primate Research Center, Atlanta, GA

\section{Corresponding Author:}

Tanja Jovanovic, Ph.D.

Grady Trauma Project

49 Jesse Hill Jr Dr NE

Atlanta, GA 30303

Phone: 404-778-1485

Email: tjovano@emory.edu 


\begin{abstract}
:
Psychophysiological measures of fear expression provide observable intermediate phenotypes of fear-related symptoms. Research Domains Criteria (RDoC) advocate using neurobiological intermediate phenotypes that provide dimensional correlates of psychopathology. Negative Valence Systems in the RDoC matrix include the construct of acute threat, which can be measured on a physiological level using potentiation of the acoustic startle reflex assessed via electromyography recordings of the orbicularis oculi muscle. Impairments in extinction of fearpotentiated startle due to high levels of fear (termed fear load) during the early phases of extinction have been observed in posttraumatic stress disorder (PTSD). The goals of the current work were to examine dimensional associations between fear-related symptoms of PTSD and fear load variables to test their validity as an intermediate phenotype. We examined extinction of fear-potentiated startle in a cohort $(n=269)$ of individuals with a broad range of civilian trauma exposure (range 0-13 traumatic events per person, mean $=3.5$ ). Based on previously reported findings, we hypothesized that fear load would be significantly associated with intrusion and fear memories of an index traumatic event. The results indicated that early extinction was correlated with intrusive thoughts $(\mathrm{p}=0.0007)$ and intense physiological reactions to trauma reminders $(\mathrm{p}=$ 0.036). Degree of adult or childhood trauma exposure, and depression severity were not associated with fear load. After controlling for age, sex, race, income, level of prior trauma, and level of fear conditioning, fear load during extinction was still significantly predictive of intrusive thoughts $(\mathrm{p}=0.004)$. The significance of these findings is that they support dimensional associations with symptom severity rather than diagnostic category and, as such, fear load may emerge as a transdiagnostic intermediate phenotype expressed across fear-related disorders (e.g., specific phobia, social phobia).
\end{abstract}


Keywords: Fear conditioning, Extinction, Startle, RDoC, PTSD, Trauma

\section{Introduction}

A primary objective of the Research Domain Criteria $(\mathrm{RDoC})$ project, initiated by the National Institute of Mental Health in 2008, is to develop psychiatric disease classifications according to the integration of observable, quantifiable neuroscientific and behavioral indices as opposed to phenomenological descriptors. This includes the identification and characterization of neurobiological intermediate phenotypes that provide dimensional correlates of psychopathology (Insel et al., 2010, Cuthbert, 2014). Ideally, these phenotypes would transcend diagnostic categories and represent fundamental neurobiological mechanisms underlying common clinical presentations of mental illness. Recent work from our group and others has explored psychophysiological measures of conditioned fear expression as an observable intermediate phenotype of fear-related symptoms common to trauma-, stressor, and anxiety-related disorders (for reviews see Johnson et al., 2012, Briscione et al., 2014).

The Negative Valence Systems in the RDoC matrix include the construct of acute threat or fear, which can be measured on a physiological level using potentiation of the acoustic startle reflex. Fear-potentiated startle is defined as the increase in the frequency or magnitude of the acoustic startle reflex in the presence of a previously neutral stimulus that has been repeatedly paired with an aversive unconditioned stimulus (Davis, 1992, Davis et al., 1993). Extinction of fear-potentiated startle has been extensively examined in recent years both in healthy populations (Norrholm et al., 2006, Norrholm et al., 2008) and in traumatized civilian (Norrholm et al., 2011) and combat (Norrholm and Jovanovic, 2011) populations. Impairments in extinction of fear- 
potentiated startle due to high levels of conditioned fear expression (termed fear load) during the early phases of extinction have been observed in posttraumatic stress disorder (PTSD; Norrholm et al., 2011). Recent studies suggest contributing factors to an individual's fear load include, but are not limited to, symptom severity in the aftermath of trauma (e.g., re-experiencing; Norrholm et al., 2011), genomics (e.g., catechol-O-methyl-transferase, COMT Val66Met polymorphism and methylation; Norrholm et al., 2013), and activational hormonal levels (e.g., estrogen; Glover et al., 2012). Fear load is also positively correlated with attention bias to threat suggesting common underlying neural circuitry (Fani et al., 2012). Although the focus of the current work was on a traumatized civilian population and the severity of their symptoms according to a selfreported PTSD scale (PTSD Symptom Scale, PSS, Falsetti et al., 1993), recent work has suggested that psychophysiological fear load may be evident in other mental disorders whose clinical presentation includes the over-expression of more complex fear behaviors (e.g., specific phobia, social phobia; Waters et al., 2014).

Fear-potentiated startle techniques have been employed in translational models of stressor- and trauma-induced behavioral responses in numerous studies across rodent and primate species (Rothbaum and Davis, 2003). This is in large part due to the conservation of the fundamental neural circuitry underlying fear expression as the model species advances phylogenetically as well as the high degree of consistency afforded by the methodologies used to observe and quantify startle responses. From a clinical perspective, empirical evidence suggests that common neural systems underlie (1) the acquisition and extinction of associations between a conditioned stimulus (CS) and an aversive unconditioned stimulus (US) with which it is paired and (2) the development, maintenance, and suppression of traumatic memories, or intrusive thoughts (Rauch et al., 2006, Jovanovic and Norrholm, 2011, VanElzakker et al., 2014). Briefly, 
for fear acquisition, these systems include the convergence of the sensory properties of a conditioned stimulus (e.g., laboratory: geometric shape; experiential: ambient environmental cues) with the aversive properties of an unconditioned stimulus (laboratory: cutaneous shock; experiential: traumatic experience) within the basolateral amygdala and subsequent projections to the central amygdala and its hypothalamic and brainstem targets (Davis, 1992). The role of the amygdala in fear conditioning and extinction has been well characterized in animal studies (Davis et al., 1995, Davis et al., 2003) and in human neuroimaging studies (Phelps et al., 2004). In addition, a very recent review by Weston (2014) highlights at least 14 human posttraumatic responses that are primarily governed by the amygdaloid complex (Weston, 2014). Further, a large body of evidence supports an inhibitory role of higher cortical regions such as the medial prefrontal cortex on amygdala-based fear expression during fear extinction in rodents and humans (Phelps et al., 2004, Quirk and Beer, 2006, Shin et al., 2006, Milad et al., 2007). Taken together, this body of empirical literature highlights the interaction between the amygdala and cortical regions such as prefrontal cortex as a conserved underlying neural system that mediates fear learning and extinction and, in clinical cases, the manifestation of quantifiable neurobiological measures.

The "neural systems" approach was discussed by Davis (1992) and later re-visited by LeDoux (2012) in an effort to guide translational and clinical investigations away the limitations of diagnostic categories and the anthropomorphizing of animal subjects and toward approaches such as that suggested by the RDoC initiative (LeDoux, 2012). For example, rather than attempting to model human mental disorders including PTSD in rodent models, LeDoux (2012) suggests the identification of common systems that are phylogenetically conserved such as those that mediate an organism's stimulus specific responses (e.g., fear rather than more generalized 
anxiety). As such, a primary objective of the current study was to examine dimensional associations between fear-related symptoms of PTSD and fear load variables to test their validity as an intermediate phenotype. Based on previously reported findings, we hypothesized that fear load would be specifically associated with intrusion and fear memories of an index traumatic event. In addition, we discuss the current results with respect to the possibility that fear load represents a transdiagnostic, core feature of fear-related disorders that is expressed across DSM5 diagnostic categories.

\section{Methods}

\subsection{Participants}

The study included 269 participants who were recruited from the general medical clinics of Grady Hospital in Atlanta, GA. Inclusion criteria were willingness to participate and ability to understand the informed consent form. Exclusion criteria included active psychosis and major medical illnesses as assessed by history and physical examinations conducted by licensed physicians. Participants were also excluded for urine toxicology that was positive for cocaine and screened for hearing impairment with an audiometer (Grason-Stadler, Model GS1710). All subjects were able to detect tones at $30 \mathrm{~dB}(\mathrm{~A}) \mathrm{SPL}$ at frequencies ranging from $250-4000 \mathrm{~Hz}$. Prior to their participation, all participants provided written informed consents approved by the Emory University Institutional Review Board and Grady Research Oversight Committee.

\subsection{Assessment}

We assessed PTSD symptoms using the Modified PTSD Symptom Scale (PSS), which is a self-report measure of symptom severity during the last two weeks on 17 items (Falsetti et al., 1993).Trauma exposure was assessed using the Traumatic Events Inventory (Gillespie et al., 
2009) which catalogs exposure to 14 criterion events, including childhood physical and sexual abuse. These instruments have been validated in this population (Binder et al., 2008, Gillespie et al., 2009).

\subsection{Fear Conditioning Paradigm}

The fear-potentiated startle protocol consisted of two phases: Fear Acquisition and Fear Extinction. The Fear Acquisition phase began with a habituation phase in which the CSs were presented without any reinforcement; the conditioning phase consisted of three blocks with four trials of each type (a reinforced conditioned stimulus, CS+; a nonreinforced conditioned stimulus, CS-; and the $40 \mathrm{~ms}, 108 \mathrm{~dB}$ noise probe alone, NA) for a total of 12 trials per block and 36 total trials. Both CSs were colored shapes presented on a computer monitor for 6 seconds. The US was a 250-ms airblast with an intensity of 140 p.s.i directed at the larynx. This US has been used in several of our previous studies (Norrholm et al., 2011) and consistently produces robust fear-potentiated startle. In all phases, the inter-trial intervals were randomized to be 9 to $22 \mathrm{sec}$ in duration. Ten minutes after the Fear Acquisition phase, participants underwent the Fear Extinction phase. The Extinction phase consisted of 6 blocks with four trials of each type (the previously reinforced CS+, CS-, and NA) for a total of 12 trials per block and 72 total trials, see Figure 1A. None of the CS presentations during Extinction was reinforced with an airblast US.

As in our previous work (Jovanovic et al., 2005, Norrholm et al., 2006, Norrholm et al., 2008, Jovanovic et al., 2009), the eyeblink component of the acoustic startle response was measured by EMG recordings of the right orbicularis oculi muscle with two 5-mm $\mathrm{Ag} / \mathrm{AgCl}$ electrodes filled with electrolyte gel. One electrode was positioned $1 \mathrm{~cm}$ below the pupil of the right eye and the other was placed $1 \mathrm{~cm}$ below the lateral canthus. Impedance levels were less 
than 6 kilo-ohms for each participant. The startle probe was a 108-dB (A) SPL, 40-ms burst of broadband noise with near instantaneous rise time, delivered binaurally through headphones.

The startle response data were acquired using the electromyography (EMG) module of the BIOPAC MP150 for Windows (Biopac Systems, Inc., Aero Camino, CA). The acquired data were filtered, rectified, and smoothed using the MindWare software suite (MindWare Technologies, Ltd., Gahanna, $\mathrm{OH}$ ) and exported for statistical analyses. The EMG signal was sampled at a frequency of $1 \mathrm{kHz}$ and filtered with low- and high-frequency cutoffs at 28 and 500 $\mathrm{Hz}$, respectively. The maximum amplitude of the eyeblink muscle contraction 20-200 ms after presentation of the startle probe was used as a measure of the acoustic startle response. As in our previous studies (e.g. Norrholm et al., 2011; Norrholm et al., 2013), we did not code low responses as zeros, but used all amplitude values.

\subsection{Data Analysis}

Fear-potentiated startle was calculated using a Difference Score by subtracting startle magnitude to the noise probe alone (NA) from startle magnitude in the presence of a CS in each conditioning block. These variables were analyzed in a mixed ANOVA with the within-subject factor of Block (3 levels for Fear Acquisition; 6 levels for Fear Extinction), trial type (2 levels, CS+ and CS-). Late Fear Acquisition was defined as block 3 of Acquisition, when discrimination learning was at maximum; Extinction was divided into 3 phases: early (blocks 1 and 2), mid (blocks 3 and 4), and late (blocks 5 and 6) extinction.

Rather than group the subjects by PTSD diagnosis, we examined the symptoms along a dimensions from low to severe in order to be consistent with the RDoC approach. We first examined correlations between continuous symptom severity and fear-potentiated startle 
variables during fear acquisition and extinction. For significant correlations (those that survived Bonferroni correction for multiple comparisons), we performed a stepwise linear regression analysis controlling for demographic information and degree of trauma exposure. Finally, in order to test for possible non-linear effects, we performed a univariate analysis of variance (ANOVA) with symptom category as the independent variable. The categorical variable was defined using the participants' responses for the items, which had 4 levels: "not at all/only once", “once per week", "2-4 times per week", and "5 or more times per week/almost always". All statistical analyses were performed in SPSS 17.0 for Windows (SPSS, Inc., Chicago, IL), with alpha set at 0.05 .

\section{Results}

\subsection{Participant Descriptives}

The participant sample was primarily African American (92.5\%) and female (66.0\%). The participants were on average 40.02 years old (range 18 to 66). The majority of participants reported being unemployed (78.5\%), with $72.5 \%$ of the sample earning less than $\$ 1000$ per

month. Table 1 shows the clinical information for the study sample. The participants were highly traumatized, with the average number of Criterion A trauma types experienced being 3.53 $(\mathrm{SD}=2.50$, range 0 to 13$)$. The average total PSS was $14.78(\mathrm{SD}=12.50)$.

\subsection{Fear Conditioning and Extinction}

A repeated measures ANOVA of Block by Trial Type (CS+, CS-) during Acquisition showed significant main effects of Block, $\mathrm{F}(2,536)=76.40$, $\mathrm{p}<0.001$, main effect of Trial Type, $\mathrm{F}(1$, 
$268)=20.13, p<0.001$, and an interaction of the two variables, $F(2,536)=21.46, p<0.001$, see

Table 2. The interaction effect was due to the discrimination between the CS+ and CS-

increasing over blocks, so that fear-potentiated startle was much higher to the CS+ than the CSduring the last two blocks, termed Late Acquisition, $F(1,268)=36.30, p<0.001$. A repeated measures ANOVA of Block by Trial Type during Extinction showed a significant main effect of Block, $\mathrm{F}(5,1330)=105.78, \mathrm{p}<0.0001$, but no main or interaction effects with Trial Type. Table 2 shows the means and standard deviations for fear-potentiated startle during Acquisition and Extinction. In order to reduce the number of variables in the subsequent analyses and focus on CS+ extinction, we collapsed the blocks into three phases (Early, Mid, Late). Fear-potentiated startle to the $\mathrm{CS}+$ showed a significant decrease during the three phases of extinction $\mathrm{F}(2$, 536) $=106.01, \mathrm{p}<0.0001$.

\subsection{Symptoms and Fear Extinction}

We examined correlations between Early, Mid and Late extinction with the three primary symptoms clusters based on the DSM-IV, i.e. Re-experiencing, Avoidance and Hyperarousal symptoms, and found that fear-potentiated startle during Early and Mid Extinction was positively correlated with Re-experiencing symptoms $(\mathrm{r}=0.12, \mathrm{p}=0.05$, and $\mathrm{r}=0.16, \mathrm{p}=0.01$, respectively). In order to further examine the fear-related symptoms, we specifically queried the association between fear extinction and the following items on the PSS: 1) frequency of intrusive distressing thoughts, 2) frequency of intense emotional upset at reminders, and 3) frequency of intense physical reaction to reminders. In addition we included the item on 4) frequency of startle response to examine the association between self-reported startle and EMG measures of fearpotentiated startle response. In order to correct for multiple comparisons (12 correlations: 3 startle variables $\mathrm{x} 4 \mathrm{PSS}$ items), we used a p=0.004 as the threshold for a significant correlation. 
Table 3 shows the correlations between these variables. As can be seen, two associations remained significant after p-value correction: frequency of intrusive distressing thoughts was correlated with Early Extinction, $r=0.21, p=0.0007$, and Mid Extinction, $r=0.20, p=0.001$. Of note, there was no association between self-reported startle and EMG startle. In order to control for demographic data and trauma history, we performed stepwise linear regressions, adding age, sex, race, and income in the first step, trauma exposure in the second step, and either Early or Mid Extinction in the final step predicting intrusive symptoms. While these covariates were not significantly associated with fear load (FPS during Early and Mid Extinction), they were included in a regression in order to see whether FPS would mediate potential associations between these variables and intrusive symptoms. The final model for Early Extinction was significant, $F(6,244)=6.35, \mathrm{p}<0.001$, and accounted for $13.8 \%$ of the variance in intrusive symptoms, with FPS alone accounting for $5.2 \%$ of the variance after controlling for demographics and trauma history, $\mathrm{F}_{\text {change }}(1,238)=14.46, \mathrm{p}<0.0001$. Similarly, the regression for Mid Extinction was significant, $\mathrm{F}(6,244)=5.79$, $\mathrm{p}<0.001$ and accounted for $12.7 \%$ of the variance in intrusive symptoms, with FPS alone accounting for $4.2 \%$ of the variance, $\mathrm{F}_{\text {change }}(1$, 238) $=11.41, \mathrm{p}=0.001$.

Given that Early Extinction is highly correlated with level of fear at the end of acquisition $(\mathrm{r}=0.46, \mathrm{p}<0.001)$, we included fear-potentiated startle to $\mathrm{CS}+$ during Late Acquisition to the model, and Early Extinction was still significantly predictive of intrusive symptoms, $\mathrm{F}_{\text {change }}(1$, $237)=12.23, p=0.001$. Of the covariates entered into the model, only female sex $(p=0.04)$ and higher trauma exposure $(\mathrm{p}<0.001)$ were predictive of higher frequencies of intrusive thoughts. Table 4 shows the beta coefficients for each variable. As shown in the table, fear-potentiated startle at the end of Acquisition did not contribute to intrusive thoughts. These results suggest 
that fear responses during extinction were associated with psychopathology above and beyond the original level of fear conditioning, i.e. the excessive fear load during extinction was not due to heightened fear conditioning. Importantly, it was also not predicted by age, race, or low income, suggesting that it may generalize to other traumatized populations.

In order to test for non-linear effects of symptom severity, we performed a univariate ANOVA comparing the participants across four categories of reported symptom frequency on FPS during extinction: "not at all/only once", "once per week", "2-4 times per week", and "5 or more times per week/almost always". Figure 1 shows FPS for each category during Early Extinction (1A) and Mid Extinction (1B). There was a significant main effect of symptom frequency category on Early Extinction, $\mathrm{F}(3,254)=4.77$, $\mathrm{p}=0.003$, and post-hoc tests indicated that the most severe category ( 5 or more times per week) was significantly higher than all other categories, $0.0002<\mathrm{p}<0.03$ (Figure 1A). While there was a main effect of category on Mid Extinction as well, $\mathrm{F}(3,254)=3.83, \mathrm{p}=0.01$, post-hoc tests showed that the most severe category differed only from the lowest frequency category, $\mathrm{p}=0.001$ (Figure $1 \mathrm{~B}$ ). These data suggest that there may be a threshold in symptom frequency in that those with 5 or more symptoms per week have higher physiological fear than those with up to 4 symptoms per week. In this sample, the extreme symptom group represented about $13 \%$ of the entire sample - these individuals have the highest fear load and resistance to extinction.

\section{Discussion}

The primary findings of the current study demonstrate that the early phase of conditioned fear extinction, during which time the fear load phenomenon is observed, is significantly correlated with intrusive thoughts $(\mathrm{p}=0.0007)$ and intense physiological reactions to trauma reminders $(\mathrm{p}=$ 0.036). In addition, the degree of adult or childhood trauma exposure was not associated with 
fear load. Further, fear load during extinction training remained significantly predictive of intrusive thoughts $(p=0.004)$ after statistically controlling for the factors of age, sex, race, income, level of prior trauma, and level of fear conditioning. The individuals with the most severe symptoms (with a frequency of 5 or more intrusive thoughts per week), had significantly greater fear-potentiated startle during early extinction than all other patients, including those who reported having symptoms 2-4 times per week, $\mathrm{p}=0.02$.

The present study extends our previous research in two important ways: first, we have further characterized the association between fear load and posttraumatic stress responses from a broad range of intrusive symptoms (e.g., Norrholm et al., 2011) to those specifically relating to fear memories of the traumatic event. The significance of this finding is that it points to dimensional associations with symptom severity rather than a diagnostic category, thereby aligning well with the RDoC approach (Insel et al., 2010). Second, rather than examining only linear relationships between symptoms and fear load, we were able to observe a non-linear threshold function indicating that high fear load is primarily present in those who are extremely symptomatic and in greatest need of treatment intervention.

The present study also replicated our earlier findings in a much larger dataset, demonstrating the robustness of the fear load phenotype in this civilian trauma population. As previously noted, we also controlled for the level of fear acquisition in the regression analysis of early extinction. Due to the high correlation between these variables, it is important to test whether extinction accounts for the variance in symptoms apart from fear acquisition. We found this to be the case; early extinction significantly predicted intrusive symptoms even after accounting for level of terminal fear acquisition. 
Recent studies have identified heterogeneous trajectories of fear extinction that represent exciting new avenues for developing targeted analytical and treatment methodologies in this area of study (Galatzer-Levy et al., 2013b). Three distinct populations have been identifying in rats based on their pattern of freezing response during extinction training. Patterns include a modal trajectory of rapid and complete fear extinction learning, a population who slowly but also completely extinguishes the learned fear response, and a minority of rats that fail to extinguish and continue to show high levels of fear in response to the conditioned stimulus (Galatzer-Levy et al., 2013b). Highly concordant patterns in PTSD symptom severity have been identified among adult trauma survivors who were followed longitudinally from immediately after trauma exposure to 14 months post-exposure (Galatzer-Levy et al., 2013a). Importantly, these analyses indicate that these are distinct phenotypes, not one population with variability in their degree of fear response.

These distinct phenotypes indicate that mechanisms and pathways, as well as sequelae in other domains of functioning may also be heterogeneous, with different causes, consequences, and treatments being relevant to different populations. For example, early psychotherapeutic interventions following trauma exposure have been shown to only have an effect for individuals in one of the three identified PTSD trajectories (Galatzer-Levy et al., 2013a). An important future direction will be to similarly empirically identify populations in traumatized individuals based on fear load. These populations may better inform our understanding of underlying neurobiological processes that lead to healthy and abnormal fear learning. Further, the effectiveness of new therapeutic interventions can be determined based on their ability to alter an individual's trajectory. 
The RDoC approach previously described (e.g., Cuthbert, 2014; Insel et al., 2010) incorporates a Negative Valence System that includes the constructs of acute threat or fear that we now suggest can be indexed through psychophysiological units of analysis within the fear learning framework. In other words, the fear load phenomenon described here and in our previous work may serve as a common feature of fear-related "psychopathology based on dimensions of observable behavior and neurobiological measures (Cuthbert, 2014)." Fear load may be an intermediate phenotype that is related to the underlying etiology of fear disorders and the symptoms of fear-related psychopathologies (Briscione et al., 2014), such as phobias (Waters et al., 2014). Although there is little transdiagnostic work in this area, some evidence suggests that fear load phenomena are present in other fear-related disorder as well. For example, highly anxious individuals with a fear of flying have displayed greater physiological arousal to both simulated and in vivo flight-related cues (Trimmel et al., 2014). In addition, elevated psychophysiological responses (including startle) have been reported in claustrophobic individuals exposed to a virtual tunnel environment (Muhlbeger et al., 2007). It remains to be seen if fear load manifests itself across fear-related psychiatric conditions including multiple classes of phobia.

Additionally, fear load can be modeled in pre-clinical research, such as the percentage of animals that have difficulty with fear extinction. Modeling fear load in animal studies is consistent with the approach advocated by LeDoux (2012) as it allows mechanistic neuroscientific investigation of this phenotype using an RDoC approach. Table 5 lists the suggested conditions that the fear load phenotype meets in order to qualify as a useful measure for RDoC studies. 
The apparent pathological feature of excessive fear load clearly represents a potential clinical target for therapeutic intervention, however, lower levels of fear may predict more favorable outcomes. As is the case with arousal and task performance, there may be an inverted U shaped curve, in that there are optimal levels of fear than can have beneficial consequences. However, if arousal levels continue to increase, performance on difficult tasks will be impaired ( for example, Yerkes-Dodson law (Diamond et al., 2007)). For example, recent investigations have explored acoustic startle responses to trauma-related stimuli (termed trauma-potentiated startle) within the presentation of a virtual reality environment (Roy et al., 2013, RobisonAndrew et al., 2014). Robison-Andrew and others (2014) showed that combat veterans who responded positively to prolonged exposure therapy (e.g., > 50\% reduction in their CAPS score over the course of treatment) displayed an initial increase in trauma-potentiated startle that was followed by a marked decrease in startle responses to trauma-related cues. It is possible that this level of fear or arousal experienced in the virtual reality session fosters engagement with the stimuli and enhanced therapeutic effects. However, once extreme levels of fear are present, they are a hindrance to fear extinction, which requires new learning (Quirk, 2002) and significant engagement of the prefrontal cortex (Milad et al., 2007) which may be hypoactive in PTSD (Milad et al., 2009). Even in psychiatrically healthy individuals, high levels of induced fear interfere with attention and cognitive processes (Moser et al., 2005). In those suffering from trauma-related disorders, fear load may represent an obstacle that must be overcome in order to achieve recovery from intrusive thoughts. In fact, in some cases, patients may self-medicate with substance abuse in order to reduce fear load, leading to further psychopathology (Davis et al., 2013). While the present research represents a sample from a low-income, primarily African American inner-city population, these demographics did not account for the observed fear load, 
wchi suggests that the findings may generalize to other traumatized populations. There is a compelling rationale to further examine fear load as an intermediate phenotype for fear-related psychopathologies. More specifically, as a biological marker of symptom severity and as a putative predictor of treatment efficacy as well as a target for therapeutic intervention. 


\section{Acknowledgments}

We thank the Grady Trauma Project staff and the nursing staff at Grady Memorial Hospital for their assistance with participant recruitment and data collection. This research was supported by National Institute of Mental Health Grants MH071537, MH096764 (PI, K.J.R.), MH098212 and MH092576 (PI, T.J.), MH10088 (PI: E.G.), the Howard Hughes Medical Institute (K.J.R.), the Atlanta Clinical Translational Science Institute, the NIH National Centers for Research Resources (M01 RR00039), and the Burroughs Wellcome Fund (K.J.R.). This work was funded in part by the Brain and Behavior Foundation (formerly NARSAD; S.D.N. and T.J.), and the Department of Defense (DOD)/Congressionally Directed Medical Research Program (CDMRP, Award \# W81XWH-08-2-0170; PI, S.D.N.). Lastly, support was provided by the VA Merit Award Program, the American Foundation for Suicide Prevention, and the American Psychoanalytic Association Psychoanalytic Research Fund (PI: B.B.).

\section{Financial Disclosures}

Drs. Norrholm, Jovanovic, Glover, Stevens, Galatzer-Levy, Fani, and Bradley report no financial disclosures. Dr. Ressler has received funding from the Burroughs Wellcome Fund, NIH, and he has an unrelated role as cofounder of Extinction Pharmaceuticals for development of $N$-methylD-aspartate- based therapeutics.

VA Acknowledgments: This material is the result of work supported with resources and the use of facilities at the Atlanta VA Medical Center, Decatur, GA. The contents of this manuscript do not represent the views of the U.S. Department of Veterans Affairs or the United States Government. The following authors are employed by the Atlanta VAMC (Decatur, GA): Dr. Seth D. Norrholm (Program Analyst, Mental Health Service Line) and Dr. Bekh Bradley (Chief of the Mental Health Service Line). 


\section{Figure and Table Captions}

Table 1. Mean, variability and range of trauma exposure and PTSD symptoms in the sample.

Table 2. Means and stardard deviations of fear-potentiated startle (FPS) responses during Acquisition and Extinction across Block and Trial Type. FPS was calculated as a differences score between startle magnitude to the CS and startle magnitude to noise alone trials.

Table 3. Correlations between individual fear-related items from the PSS and fear extinction.

Table 4. Association between predictors in regression analyses with intrusive symptoms.

Table 5. List of conditions that are met by the fear load phenotype of relevance to RDoC

Figure 1. Fear-potentiated startle during A) Early extinction (blocks 1-2) and B) Mid Extinction (blocks 3-4) across the different levels of responses on the "intrusive distressing thoughts" item on the PSS. PSS=PTSD Symptom Scale 


\section{References}

Binder EB, Bradley RG, Liu W, Epstein MP, Deveau TC, Mercer KB, Tang Y, Gillespie CF, Heim CM, Nemeroff CB, Schwartz AC, Cubells JF, Ressler KJ (2008) Association of FKBP5 Polymorphisms and Childhood Abuse With Risk of Posttraumatic Stress Disorder Symptoms in Adults. JAMA 299:1291-1305.

Briscione MA, Jovanovic T, Norrholm SD (2014) Conditioned Fear Associated Phenotypes as Robust, Translational Indices of Trauma-, Stressor-, and Anxiety-related Behaviors. Frontiers in Psychiatry 5.

Cuthbert BN (2014) The RDoC framework: facilitating transition from ICD/DSM to dimensional approaches that integrate neuroscience and psychopathology. World Psychiatry 13:28-35.

Davis M (1992) The role of the amygdala in conditioned fear. In: The Amygdala: Neurobiological Aspects of Emotion, Memory and Mental Dysfunction (Aggleton, J., ed), pp 255-305 New York: Wiley-Liss.

Davis M, Falls WA, Campeau S, Kim M (1993) Fear-potentiated startle: A neural and pharmacological analysis. Behavioral Brain Research 58:175-198.

Davis M, McNish K, Gewirtz J, Kim M (1995) The roles of the amygdala and bed nucleus of the stria terminalis (BNST) in the acquisition of fear-potentiated startle using both explicit and contextual cues. Society for Neuroscience Abstracts 21:1224.

Davis M, Walker DL, Myers KM (2003) Role of the amygdala in fear extinction measured with potentiated startle. Ann N Y Acad Sci 985:218-232.

Davis TA, Jovanovic T, Norrholm SD, Glover EM, Swanson M, Spann S, Bradley B (2013) Substance Use Attenuates Physiological Responses Associated With PTSD among Individuals with Co-Morbid PTSD and SUDs. J Psychol Psychother Epub.

Diamond DM, Campbell AM, Park CR, Halonen J, Zoladz PR (2007) The temporal dynamics model of emotional memory processing: a synthesis on the neurobiological basis of stress-induced amnesia, flashbulb and traumatic memories, and the Yerkes-Dodson law. Neural Plast 2007:60803.

Falsetti S, Resnick H, Resick P, Kilpatrick D (1993) The Modified PTSD Symptom Scale: A brief self-report measure of posttraumatic stress disorder. The Behavior Therapist 16:161-162.

Fani N, Tone EB, Phifer J, Norrholm SD, Bradley B, Ressler KJ, Kamkwalala A, Jovanovic T (2012) Attention bias toward threat is associated with exaggerated fear expression and impaired extinction in PTSD. Psychological Medicine 42:533-543. .

Galatzer-Levy IR, Ankri Y, Freedman S, Israeli-Shalev Y, Roitman P, Gilad M, Shalev AY (2013a) Early PTSD symptom trajectories: persistence, recovery, and response to treatment: results from the Jerusalem Trauma Outreach and Prevention Study (J-TOPS). PLoS One 8:e70084.

Galatzer-Levy IR, Bonanno GA, Bush DE, LeDoux J (2013b) Heterogeneity in Threat Extinction Learning: Substantive and Methodological Considerations for Identifying Individual Difference in Response to Stress. Frontiers in Behavioral Neuroscience 7.

Gillespie CF, Bradley RG, Mercer K, Smith AK, Conneely K, Gapen M, Weiss T, Schwartz AC, Cubells JF, Ressler KJ (2009) Trauma Exposure and Stress-Related Disorders in Inner City Primary Care Patients. General Hospital Psychiatry 31:505-514.

Glover EM, Jovanovic T, Mercer KB, Kerley K, Bradley B, Ressler KJ, Norrholm SD (2012) Estrogen Levels Are Associated with Extinction Deficits in Women with Posttraumatic Stress Disorder. Biological Psychiatry 72:19-24. 
Insel T, Cuthbert B, Garvey M, Heinssen R, Pine DS, Quinn K, Sanislow C, Wang P (2010) Research domain criteria (RDoC): toward a new classification framework for research on mental disorders. Am J Psychiatry 167:748-751.

Johnson LR, McGuire J, Lazarus R, Palmer AA (2012) Pavlovian fear memory circuits and phenotype models of PTSD. Neuropharmacology 62:638-646.

Jovanovic T, Keyes M, Fiallos A, Myers KM, Davis M, Duncan E (2005) Fear Potentiation and Fear Inhibition in a Human Fear-Potentiated Startle Paradigm. Biological Psychiatry 57:1559-1564.

Jovanovic T, Norrholm SD (2011) Neural mechanisms of impaired fear inhibition in posttraumatic stress disorder. Frontiers in Behavioral Neuroscience 5.

Jovanovic T, Norrholm SD, Fennell JE, Keyes M, Fiallos AM, Myers KM, Davis M, Duncan EJ (2009) Posttraumatic stress disorder may be associated with impaired fear inhibition: Relation to symptom severity. Psychiatry Research 167:151-160.

LeDoux J (2012) Rethinking the Emotional Brain. Neuron 73:653-676.

Milad MR, Pitman RK, Ellis CB, Gold AL, Shin LM, Lasko NaB, Zeidan MA, Handwerger K, Orr SP, Rauch SL (2009) Neurobiological Basis of Failure to Recall Extinction Memory in Posttraumatic Stress Disorder. Biological Psychiatry 66:1075-1082.

Milad MR, Wright CI, Orr SP, Pitman RK, Quirk GJ, Rauch SL (2007) Recall of fear extinction in humans activates the ventromedial prefrontal cortex and hippocampus in concert. Biol Psychiatry 62:446-454.

Moser JS, Hajcak G, Simons RF (2005) The effects of fear on performance monitoring and attentional allocation. Psychophysiology 42:261-268.

Mühlberger A, Bülthoff H, Wiedemann G, Pauli P (2007). Virtual reality for the psychophysiological assessment of phobic fear: Responses during virtual tunnel driving. Psychological Assessment Vol 19: 340-346.Norrholm SD, Jovanovic T (2011) Translational Fear Inhibition Models as Indices of Trauma-Related Psychopathology. Current Psychiatry Reviews in press.

Norrholm SD, Jovanovic T, Olin IW, Sands LA, Karapanou I, Bradley B, Ressler KJ (2011) Fear extinction in traumatized civilians with posttraumatic stress disorder: relation to symptom severity. Biol Psychiatry 69:556-563.

Norrholm SD, Jovanovic T, Smith A, Binder EB, Klengel T, Conneely K, Mercer KB, Davis JS, Kerley K, Winkler JA, Gillespie CF, Bradley B, Ressler KJ (2013) Differential Genetic and Epigenetic Regulation of Catechol-O-Methyl-Transferase (COMT) is Associated with Impaired Fear Inhibition in Posttraumatic Stress Disorder. Frontiers in Behavioral Neuroscience 7.

Norrholm SD, Jovanovic T, Vervliet B, Myers KM, Davis M, Rothbaum BO, Duncan EJ (2006) Conditioned fear extinction and reinstatement in a human fear-potentiated startle paradigm. Learning \& Memory 13:681-685.

Norrholm SD, Vervliet B, Jovanovic T, Boshoven W, Myers KM, Davis M, Rothbaum B, Duncan EJ (2008) Timing of extinction relative to acquisition: a parametric analysis of fear extinction in humans. Behav Neurosci 122:1016-1030.

Phelps EA, Delgado MR, Nearing KI, LeDoux JE (2004) Extinction Learning in Humans: Role of the Amygdala and vmPFC. Neuron 43:897-905.

Quirk GJ (2002) Memory for extinction of conditioned fear is long-lasting and persists following spontaneous recovery.[see comment]. Learning \& Memory 9:402-407. 
Quirk GJ, Beer JS (2006) Prefrontal involvement in the regulation of emotion: convergence of rat and human studies. Current Opinion in Neurobiology 16:723-727.

Rauch SL, Shin LM, Phelps EA (2006) Neurocircuitry Models of Posttraumatic Stress Disorder and Extinction: Human Neuroimaging Research-Past, Present, and Future. Biological Psychiatry 60:376-382.

Robison-Andrew EJ, Duval ER, Nelson CB, Echiverri-Cohen A, Giardino N, Defever A, Norrholm SD, Jovanovic T, Rothbaum BO, Liberzon I, Rauch SAM (2014) Changes in trauma-potentiated startle with treatment of posttraumatic stress disorder in combat Veterans. Journal of Anxiety Disorders 28:358-362.

Rothbaum BO, Davis M (2003) Applying learning principles to the treatment of post-trauma reactions. Annals of the New York Academy of Sciences 1008:112-121.

Roy MJ, Costanzo ME, Jovanovic T, Leaman S, Taylor P, Norrholm SD, Rizzo AA (2013) Heart rate response to fear conditioning and virtual reality in subthreshold PTSD. Stud Health Technol Inform 191:115-119.

Shin LM, Rauch SL, Pitman RK (2006) Amygdala, Medial Prefrontal Cortex, and Hippocampal Function in PTSD. Ann NY Acad Sci 1071:67-79.

Trimmel M, Burger M, Langer G, Trimmel K (2014) Treatment of Fear of Flying: Behavioral, Subjective, and Cardiovascular Effects. Aviation, Space, and Environmental Medicine 85:550-562.

VanElzakker MB, Kathryn Dahlgren M, Caroline Davis F, Dubois S, Shin LM (2014) From Pavlov to PTSD: The extinction of conditioned fear in rodents, humans, and anxiety disorders. Neurobiology of learning and memory 113:3-18.

Waters AM, Nazarian M, Mineka S, Zinbarg RE, Griffith JW, Naliboff B, Ornitz EM, Craske MG (2014) Context and explicit threat cue modulation of the startle reflex: Preliminary evidence of distinctions between adolescents with principal fear disorders versus distress disorders. Psychiatry Research 217:93-99.

Weston CS (2014) posttraumatic stress disorder: a theoretical model of the hyperarousal subtype. Frontiers in Psychiatry 5. 


\begin{tabular}{lcccc}
\hline & Mean & SD & Minimum & Maximum \\
\hline Trauma Exposure & 3.53 & 2.50 & 0.00 & 13.00 \\
Re-experiencing Symptoms & 3.62 & 3.84 & 0.00 & 15.00 \\
Avoidance Symptoms & 5.92 & 5.59 & 0.00 & 21.00 \\
Hyperarousal Symptoms & 5.27 & 4.49 & 0.00 & 15.00 \\
\hline
\end{tabular}




\begin{tabular}{|l|cc|cc|}
\hline \multirow{2}{*}{ BLOCK } & \multicolumn{4}{|c|}{ TRIAL TYPE } \\
\cline { 2 - 5 } & \multicolumn{2}{|c|}{ CS+ } & \multicolumn{2}{c|}{ CS- } \\
\cline { 2 - 5 } ACQ 1 & M $(\mu \mathrm{V})$ & SD & $\mathrm{M}(\mu \mathrm{V})$ & SD \\
\cline { 2 - 5 } ACQ 2 & 47.48 & 64.94 & 59.06 & 84.85 \\
ACQ 3 & 22.89 & 58.55 & 3.33 & 40.72 \\
& & & 30.24 & 55.21 \\
EXT 1 & 56.49 & 74.23 & 53.62 & 75.90 \\
EXT 2 & 35.16 & 60.07 & 31.36 & 65.65 \\
EXT 3 & 19.79 & 40.09 & 18.22 & 48.68 \\
EXT 4 & 14.81 & 46.37 & 9.35 & 38.24 \\
EXT 5 & 2.84 & 36.83 & 3.38 & 29.28 \\
EXT 6 & -0.28 & 25.01 & -1.19 & 28.93 \\
\hline
\end{tabular}




\begin{tabular}{c|ccc}
\hline CORRELATIONS & Early Extinction & Mid Extinction & Late Extinction \\
\hline $\begin{array}{c}\text { intrusive/distressing } \\
\text { thoughts }\end{array}$ & $r=0.255$ & $r=0.202$ & $r=-0.003$ \\
\hline $\begin{array}{c}\text { intense emot upset at } \\
\text { reminder }\end{array}$ & $r=0.078$ & $p=0.001$ & $p=0.958$ \\
\hline $\begin{array}{c}\text { intense phys rxns at } \\
\text { reminder }\end{array}$ & $\mathrm{p}=0.215$ & $\mathrm{p}=0.122$ & $\mathrm{r}=0.097$ \\
& $\mathrm{p}=0.036$ & $\mathrm{r}=0.168$ & $\mathrm{p}=0.901$ \\
\hline jumpier / easily startled & $\mathrm{r}=0.086$ & $\mathrm{p}=0.007$ & $\mathrm{p}=0.599$ \\
& $\mathrm{p}=0.173$ & $\mathrm{r}=0.072$ & $\mathrm{r}=0.083$ \\
& & $\mathrm{p}=0.254$ & $\mathrm{p}=0.187$ \\
\hline
\end{tabular}


Outcome: Frequency of Intrusive Distressing Thoughts

\begin{tabular}{|lccc|}
\hline Standardized Coefficients & $\boldsymbol{\beta}$ & $\mathbf{t}$ & $\mathbf{p}$ \\
\hline Predictors: & & & \\
\cline { 1 - 2 } & 0.04 & 0.72 & 0.47 \\
Sex (male=0, female=1) & 0.13 & 2.06 & $\mathbf{0 . 0 4}$ \\
Race & 0.00 & -0.07 & 0.94 \\
Income & -0.02 & -0.35 & 0.73 \\
Trauma Exposure & 0.29 & 4.60 & $\mathbf{0 . 0 0 0 0 1}$ \\
Late Acquisition CS+ FPS & -0.03 & -0.42 & 0.68 \\
Early Extinction CS+ FPS & 0.25 & 3.50 & $\mathbf{0 . 0 0 1}$ \\
\hline
\end{tabular}




\section{CONDITION}

V RDoC construct (Acute Threat/Fear)

V Defined neural circuitry

Associated with symptom severity/clinical

V symptoms

V Dimensional measure

V Non-invasive measurement

V Can be tested across different human populations

V Can be modeled in animals 

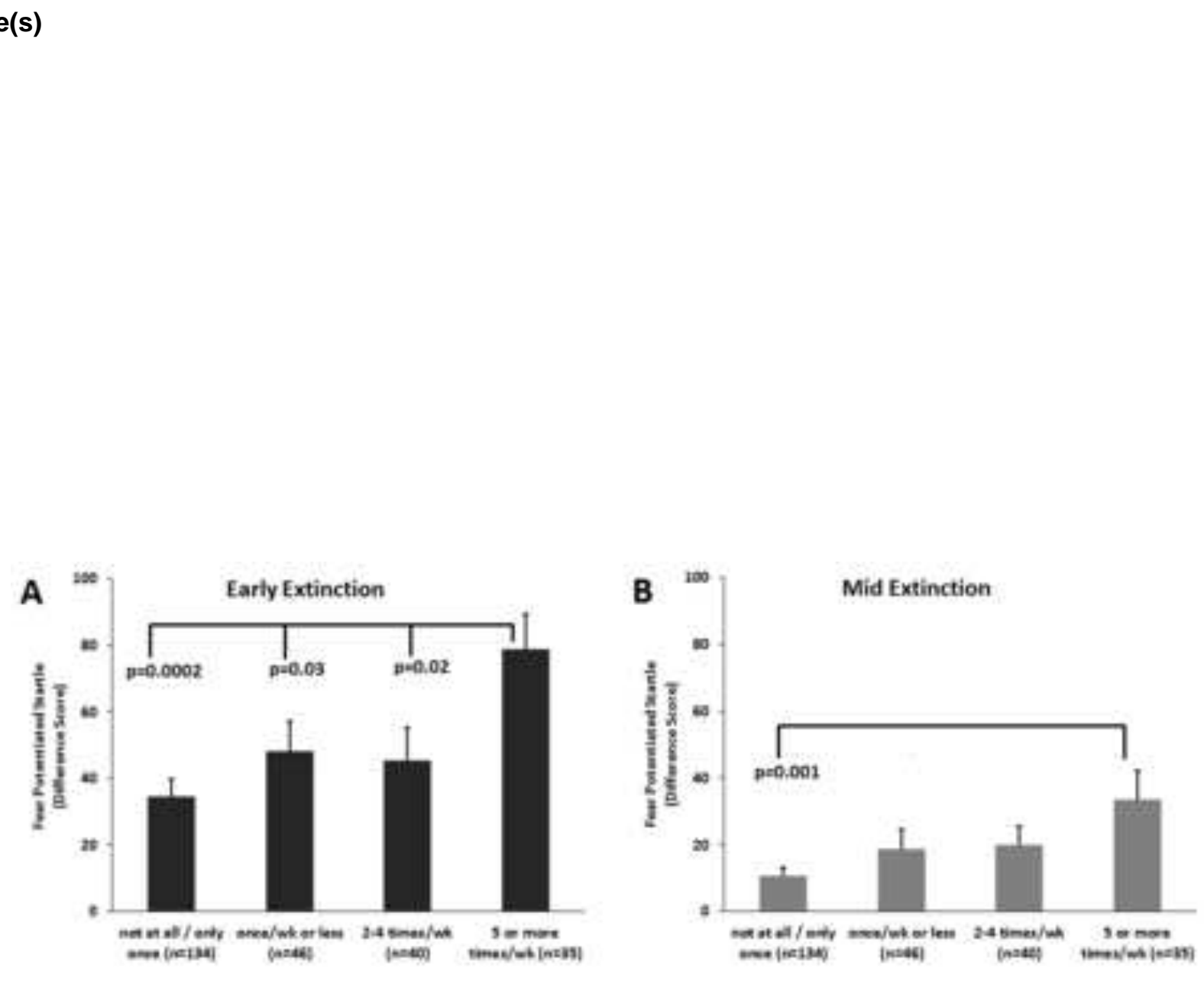